\title{
Comment on: Nathan DM, Buse JB, Davidson MB et al (2006) Management of hyperglycaemia in type 2 diabetes: a consensus algorithm for the initiation and adjustment of therapy. A consensus statement from the American Diabetes Association and the European Association for the Study of Diabetes. Diabetologia 49:1711-1721
}

\author{
E. Cerasi
}

Received: 21 November 2006 / Accepted: 21 November 2006 / Published online: 23 December 2006

(C) Springer-Verlag 2006

To the Editor: The past decade has witnessed the introduction of numerous novel therapeutic agents for type 2 diabetes, from thiazolidinediones to engineered insulins, not to mention the incretin-based therapeutics that are on the verge of entering the market. Confronted with so many options, many physicians could feel confused and unsure regarding the choice of therapeutic agent. It may therefore be understandable that the American Diabetes Association and the European Association for the Study of Diabetes have judged it necessary to generate a consensus statement on the subject, presented simultaneously in Diabetologia and Diabetes Care by Nathan et al. [1, 2]. Nevertheless, I regret that these two societies, which together exert the strongest influence on world diabetes opinion, decided to publish a consensus algorithm, because I believe that diabetes treatment is too complex to be reduced to a useful algorithm.

The heterogeneity of type 2 diabetes is textbook knowledge; diabetic patients are extremely variable regarding their phenotype, disease course, response to treatment, etc., even within homogeneous populations, and different cultures and ethnic backgrounds compound variability. Unfortunately, no single therapeutic agent has the ability

E. Cerasi $(\bowtie)$

Department of Endocrinology and Metabolism,

Hadassah University Hospital,

91120 Jerusalem, Israel

e-mail: Erol.Cerasi@huji.ac.il to normalise metabolism in type 2 diabetes. With all due respect to the outstanding science behind the development of the thiazolidinediones, they have not even matched the efficacy of age-old drugs such as metformin and sulfonylurea. Therefore, type 2 diabetes therapy continues to very much rely on the experience of the physician, which mainly translates into his ability to identify the right combination of drugs, diet and lifestyle for the individual patient, and his persuasive capacity to have patients adhere to recommendations over the years.

To whom does the consensus statement address itself? Certainly not to the diabetes specialist. The authors state that 'the algorithm that we propose is likely to engender debate,' and that 'there was no strong consensus regarding the second medication added after metformin.' I am convinced there will be even less consensus outside the restricted group of specialists who formulated this 'consensus' (sic) statement. Is it for the general practitioner? The decision tree in Fig. 2 suggests the addition of basal insulin, sulfonylurea or glitazone ad libitum if lifestyle modification plus metformin is not sufficient. This is a big help indeed to the non-diabetes specialist! Not to mention the recommendation of intensified insulin treatment-not many of the general practitioners I know would like to assume this responsibility without first consulting a diabetes specialist. I am all for transferring much of the responsibility of diabetes care to general practitioners, provided it is done under the guidance of, and involves continuous interaction with, diabetes specialists. I am strongly against distributing a printed list of recommendations and hoping for the best. 
I also wish to take issue with specific points in the recommendations. The initiation of drug treatment with metformin is given as an absolute rule. This may be logical for the USA, which has a greater proportion of hyper-obese patients among its diabetic population, but is this necessarily correct elsewhere? I and many of my European colleagues have used sulfonylurea as first drug in scores of patients with success, as we have metformin. Furthermore, a recent study in a very large patient population in Japan reported that $\sim 40 \%$ of patients were treated with sulfonylurea as single drug with excellent effect, with these patients achieving a mean $\mathrm{HbA}_{1 \mathrm{c}}$ of 7.1 [3]. The risk of severe hypoglycaemia is very small with modern sulfonylureas, and there appears to be no evidence to justify the fear of cardiovascular events related to sulfonylurea treatment $[4,5]$. As for the weight gain of $\sim 2 \mathrm{~kg}$, is it not mainly the result of correction of glucosuria and/or lack of adherence to diet? On what basis do the authors state that this 'may have an adverse impact on cardiovascular risk'? I would be much more concerned by the link between glitazone and heart failure! Finally, a sobering observation in a recent study from the USA: $25 \%$ of patients treated with metformin and/or glitazones had a potential contraindication (usually renal or cardiac) to these drugs [6].

To finish on a positive note: this consensus statement once again draws attention to the necessity to act rapidly and drastically in type 2 diabetes for achievement of nearnormal glycaemic control. The era of 'wait and see,' with half-year periods between each therapeutic step, is hopefully gone forever. Type 2 diabetic patients are just as entitled to intensified treatment as type 1 diabetic patients are.

\section{References}

1. Nathan DM, Buse JB, Davidson MB et al (2006) Management of hyperglycaemia in type 2 diabetes: a consensus algorithm for the initiation and adjustment of therapy. A consensus statement from the American Diabetes Association and the European Association for the Study of Diabetes. Diabetologia 49:1711-1721

2. Nathan DM, Buse JB, Davidson MB et al (2006) Management of hyperglycemia in type 2 diabetes: a consensus algorithm for the initiation and adjustment of therapy. A consensus statement from the American Diabetes Association and the European Association for the Study of Diabetes. Diabetes Care 29:1963-1972

3. Kobayashi M, Yamazaki K, Hirao K et al (2006) The status of diabetes control and antidiabetic drug therapy in Japan-a crosssectional survey of 17,000 patients with diabetes mellitus (JDDM 1). Diabetes Res Clin Pract 73:198-204

4. Leibowitz G, Cerasi E (1996) Sulfonylurea treatment of NIDDM patients with cardiovascular disease: a mixed blessing? Diabetologia 39:503-514

5. UK Prospective Diabetes Study (UKPDS) Group (1998) Intensive blood glucose control with sulphonylureas or insulin compared with conventional treatment and risk of complication in patients with type 2 diabetes (UKPDS 33). Lancet 352:837-853

6. MacLean CD, Littenberg B, Kennedy AG (2006) Limitations of diabetes pharmacotherapy: results from the Vermont Diabetes Information System study. BMC Fam Pract 7:50 